\title{
PET/MRI, Part 2: Technologic Principles
}

\author{
Geoffrey M. Currie ${ }^{1,2}$, Peter Kamvosoulis ${ }^{3}$, and Stewart Bushong ${ }^{2}$ \\ ${ }^{1}$ School of Dentistry and Health Science, Charles Sturt University, Wagga Wagga, Australia; ${ }^{2}$ Department of Radiology, Baylor College of \\ Medicine, Houston, Texas; and ${ }^{3}$ Magnetic Resonance Department, New York-Presbyterian/Weill Cornell Medical Center, New York, \\ New York
}

\begin{abstract}
CE credit: For CE credit, you can access the test for this article, as well as additional JNMT CE tests, online at https://www.snmmilearningcenter.org. Complete the test online no later than September 2024. Your online test will be scored immediately. You may make 3 attempts to pass the test and must answer $80 \%$ of the questions correctly to receive $1.0 \mathrm{CEH}$ (Continuing Education Hour) credit. SNMMI members will have their CEH credit added to their VOICE transcript automatically; nonmembers will be able to print out a CE certificate upon successfully completing the test. The online test is free to SNMMI members; nonmembers must pay $\$ 15.00$ by credit card when logging onto the website to take the test.
\end{abstract}

The challenges of hybridizing PET and MRI as a simultaneous modality have been largely overcome in recent times. PET hybridized with MRI has seen the emergence of PET/MRI systems in the clinical setting, and with it comes a responsibility to adapt appropriate facility design, safety practices, protocols and procedures, and clinical opportunity. This article provides an insight into the considerations and challenges associated with PET/MR technology. Given that the nature of PET is well established among the readership of this journal, the article provides an introduction to the foundations of $\mathrm{MRI}$ instrumentation and emphasis on specific technologic aspects of PET/MR systems. This article is the second in a 4-part integrated series sponsored by the PET/MR and Publication Committees of the Society of Nuclear Medicine and Molecular Imaging-Technologist Section, building on the previous article (part 1), which was on establishing a facility. In subsequent parts, PET/ MRI will be explored on the basis of protocols and procedures (part 3) and applications and clinical cases (part 4).

Key Words: PET/MR; PET; MRI; PET/MRI

J Nucl Med Technol 2021; 49:217-225

DOI: $10.2967 /$ jnmt.120.261862

\section{A}

lthough the merits of SPECT, PET, CT and MRI have been widely recognized and reported in both research and clinical practice, the synergistic hybridization of imaging modalities has revolutionized diagnostic imaging. SPECT/ $\mathrm{CT}$ and PET/CT are in widespread practice, merging the advantages of the high-resolution anatomic information of the CT with the physiologic and molecule-level functional information from the nuclear medicine modalities. More recently, PET/MRI hybrid systems have emerged, although PET/MR was devised and patented in the 1990s (1). The first integrated PET/MRI systems emerged in 2010, and by 2015 there were about 110 PET/MRI systems globally $(\sim 160$ in

\footnotetext{
Received Dec. 19, 2020; revision accepted Feb. 11, 2021.

For correspondence or reprints, contact Geoffrey M. Currie (gcurrie@ csu.edu.au).

Published online March 15, 2021.

COPYRIGHT (C) 2021 by the Society of Nuclear Medicine and Molecular Imaging
}

2020), with Siemens representing nearly $80 \%$ of the market, ahead of Philips and GE Healthcare (2). For clarification and consistency, the terms PET/MR and PET/MRI are not used interchangeably in this article. $P E T / M R$ is used in reference to the technology and principles, whereas PET/MRI refers to the application of that technology in imaging. The use of MRI versus MR throughout should be self-explanatory. Furthermore, the term imaging system is used in preference to the more archaic use of the term scanner.

A working knowledge of PET is assumed in this discussion but can be refreshed in the previously published work of Turkington (3). The general principles of MRI are outlined below. This is also not a forum for debating the relative merits of PET/MRI against PET/CT. Nonetheless, it would be remiss not to highlight the motivations for PET/MRI $(1,4-7)$. First, PET/MRI produces a reduced patient radiation dose. This is particularly important in pediatric patients, women of childbearing age, and the numerous patients who undergo sequential scanning to monitor response to therapy or recurrence. Compared with PET/CT, PET/MRI can reduce the dose to just $25 \%$, given that $75 \%$ of the patient radiation dose is associated with CT. An example would be the reduction in a pediatric whole-body PET/CT scan from 19.5 to 4.6 $\mathrm{mSv}$ for PET/MRI. Second, soft-tissue visualization and contrast on MRI are superior to those on CT, enhancing lesion detection and diagnostic accuracy in numerous conditions. MRI adds important complementary functional information to the PET scan, further enhancing outcomes. The opportunity from PET/MRI for multiparametric imaging increases radiomic feature extraction and diagnostic utility. Third, PET/MR offers genuinely simultaneous imaging, which has a positive impact on coregistration, especially in organs that move (e.g., heart and lungs), in lesions that experience physiologic motion (e.g., lung tumors), in organs that change (e.g., filling of the urinary bladder), or in patients who move during scanning (e.g., from pain or anxiety). The simultaneous imaging also allows better synchronization of respiratory and cardiac gating between PET and MRI data, as well as allowing application of motion correction to the PET data. The correction for motion increases lesion detection and quantitative 
accuracy. Fourth, there is also a reported efficiency when PET and MRI are required, because they are acquired simultaneously. In the absence of PET/MRI, PET/CT is sequential and then requires the additional time of the stand-alone MRI. Finally, image reconstruction offers the chance to correct partial-volume artifacts on the PET images using the MRI coregistration. The net impact of this approach is resolution recovery or enhanced spatial resolution of the PET data.

Despite these advantages, replacing PET/CT with PET/MR is associated with several challenges, such as detector function in a magnetic field, attenuation correction, scatter correction, artifacts, and truncation of the field of view. These challenges are especially true when one is considering truly integrated PET/MR with simultaneous acquisition $(1,4,5,7)$.

\section{FOUNDATIONS OF MRI}

For the MRI novice, the basic foundations allow a working knowledge of the principles of MRI. For some, the superficial introduction outlined below will provide sufficient insight. Others will yearn for deeper understanding beyond the scope and word limit of this article. Such insight can be sourced from several key textbooks $(8,9)$. Rather than exploring Newtonian and quantum physics, a simpler model of MRI is offered.

\section{MR Signal}

Some nuclei spin on their own axis, which allows them to be thought of as small magnets. Hydrogen is one such nuclei-indeed, the one that produces the strongest magnet. Consider the distribution of hydrogen in the human body. It is clear that hydrogen imaging would be both biologically useful for some tissues and sufficiently abundant in distribution to allow quality imaging. The dispersion of hydrogen in objects is random and fairly uniform, which means the small magnets in opposite alignments cancel one another out to produce a net zero magnetic vector (Fig. 1A). If a magnetic field is applied to the object, then the hydrogen atoms align parallel with the magnetic field and the object has a net magnetic vector; it has become polarized (Fig. 1B) (8-10). In reality, not all

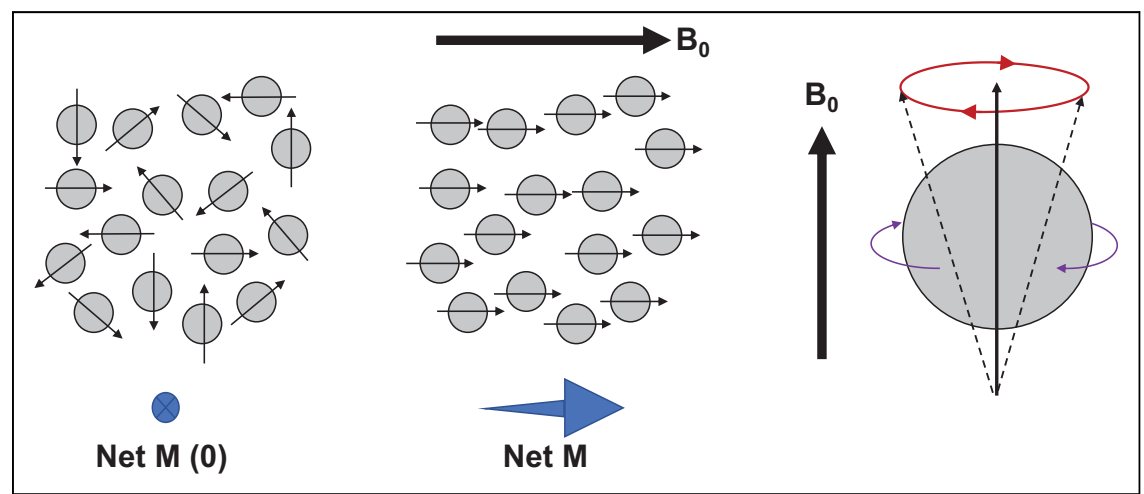

FIGURE 1. Nucleus spin creates polarity with random distribution, producing net magnetic vector $(\mathrm{M})$ of zero (left). Application of strong magnetic field $\left(\mathrm{B}_{0}\right)$ creates alignment of proton dipoles, producing net $\mathrm{M}$ aligned with $\mathrm{B}_{0}$ (middle). Although proton dipoles spin on their axis (purple arrows) to produce small magnets, movement in presence of magnetic field is gyromagnetic (red arrows) and termed precession (right). hydrogen atoms (let us refer to them as proton dipoles) become aligned with the magnetic field. Approximately 1 per million of proton dipoles realign with the magnetic field, but this is sufficient to create polarization (8). Although a simple schematic representation such as Figure 1 suggests that the atoms have aligned uniformly, in reality they wobble. The wobble is known as precession (Fig. 1C), and the frequency of the precession is called the Larmor frequency and depends on the strength of the magnetic field $(8-10)$. For hydrogen, a 1.5-T MRI system produces $63.9 \mathrm{MHz}$ of precession whereas $3 \mathrm{~T}$ produces $127.8 \mathrm{MHz}$ of precession $(8,9)$. Indeed, this might be better expressed as a gyromagnetic ratio of $\mathrm{MHz}$ of precession per $\mathrm{T}$. This is important because each dipole nucleus has a unique gyromagnetic ratio. For hydrogen $\left({ }^{1} \mathrm{H}\right)$ it is $42.6 \mathrm{MHz} / \mathrm{T}$, whereas others include ${ }^{19} \mathrm{~F}$ at $40.1 \mathrm{MHz} / \mathrm{T},{ }^{31} \mathrm{P}$ at $17.2 \mathrm{MHz} / \mathrm{T},{ }^{23} \mathrm{Na}$ at $11.3 \mathrm{MHz} /$ $\mathrm{T}$, and ${ }^{13} \mathrm{C}$ at $10.7 \mathrm{MHz} / \mathrm{T}$.

\section{Excitation}

Consider the patient in an MRI system. In the presence of the magnetic field, proton dipoles align with the magnetic field to produce a net magnetic vector (Fig. 1B). This is also referred to as the equilibrium magnetization state. The magnetic field causes each dipole to randomly precess (Fig. 2A). If a pulse of radiofrequency is emitted at the Larmor frequency, it will change the energy state of the hydrogen nuclei (excitation) (8-10). This is achieved using the radiofrequency coil, which pulses at resonance and causes the net magnetic vector to flip to negative on the $z$-axis. Despite this, the precession remains perpendicular to the $z$-axis, but instead of being random, the precession of each dipole becomes in phase (Fig. 2B) (8-10). This is referred to as phase coherence and generates the optimal MR signal. The net magnetism returns to the equilibrium magnetism state (relaxation) $(8-10)$. This creates a moving magnetic field, and electric current can then be generated in a radiofrequency receiver coil (Fig. 2C) $(8-10)$. The time for the signal to return to equilibrium is called relaxation time, and the signal produced is referred to as free induction decay (FID) $(8-10)$.

\section{Relaxation}

Relaxation time is associated with 2 independent processes, $\mathrm{T}_{1}$ and $\mathrm{T}_{2}$, that occur simultaneously $(8-10)$. $\mathrm{T}_{1}$ relaxation is also known as longitudinal relaxation because it describes the rate at which the magnetism returns to equilibrium. $\mathrm{T}_{2}$ relaxation is also known as transverse relaxation because it describes the rate at which phasecoherent precession dephases. $\mathrm{T}_{1}$ is the time required to return to $63 \%$ of the equilibrium value after exposure to a $90^{\circ}$ radiofrequency pulse $(8-10)$. Conversely, $\mathrm{T}_{2}$ is the time required to 

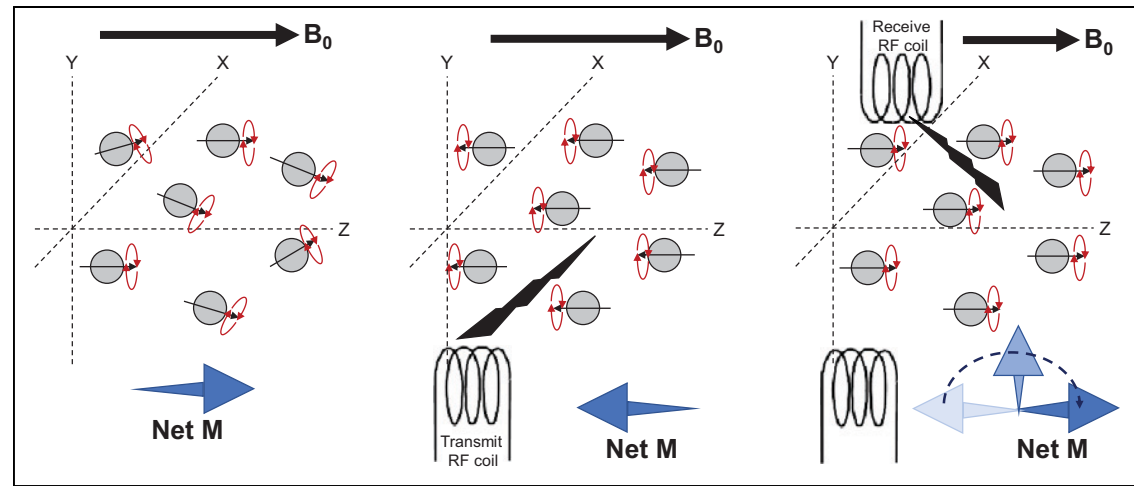

FIGURE 2. Nucleus spin creates polarity with magnetic field $\left(\mathrm{B}_{0}\right)$ and positive alignment of proton dipoles with $z$-axis, producing net magnetic vector $(\mathrm{M})$ and random precession (left). Radiofrequency (RF) transmission causes aligned proton dipoles to flip to negative on $z$-axis and causes precession to come into phase (middle). Relaxation results in reversion of net M, producing FID signal at RF receiver coil (right).

dephase the signal to $37 \%$ of its original value. The role of contrast medium is to alter the relaxation times (Fig. 3) (8-10). Relaxivity measures the degree to which a given amount of contrast agent shortens $\mathrm{T}_{1}$ or $\mathrm{T}_{2}$, which means higher relaxivity produces better enhancement (11).

\section{Image Formation}

The FID signal intensity decreases over time as a harmonic oscillation (Fig. 4A) (8). Figure 4A represents signal intensity over time, but this can be converted to the frequency domain via Fourier transformation to produce a nuclear MR spectrum
(Fig. 4B). The Fourier transformation of the measured MRI signal is referred to as $k$-space. The clusters of signals at different frequencies represent the specific nuclear MR characteristics of different tissues (Fig. 4C) (8-10). Although this provides an insight into the proportion of various tissues in a sample (as used in nuclear MR spectroscopy), it does not provide information about the location of those tissues. Superimposed on the static magnetic field, a gradient magnetic field is applied with different field strengths, and this allows tissue localization (Fig. 4D) (8-10). A far more complex FID is produced by data collected at multiple projections, which can then be reconstructed using tomographic principles previously described (12).

\section{Pulse Sequences}

Although this provides a simple explanation of the MRI process, in reality, FID is not a simple imaging option because of the weak signal (8-10). A 1-pulse sequence would need to be repeated several hundreds of times to generate an adequate signal $(8,9)$. The time between each radiofrequency pulse is called the repetition time
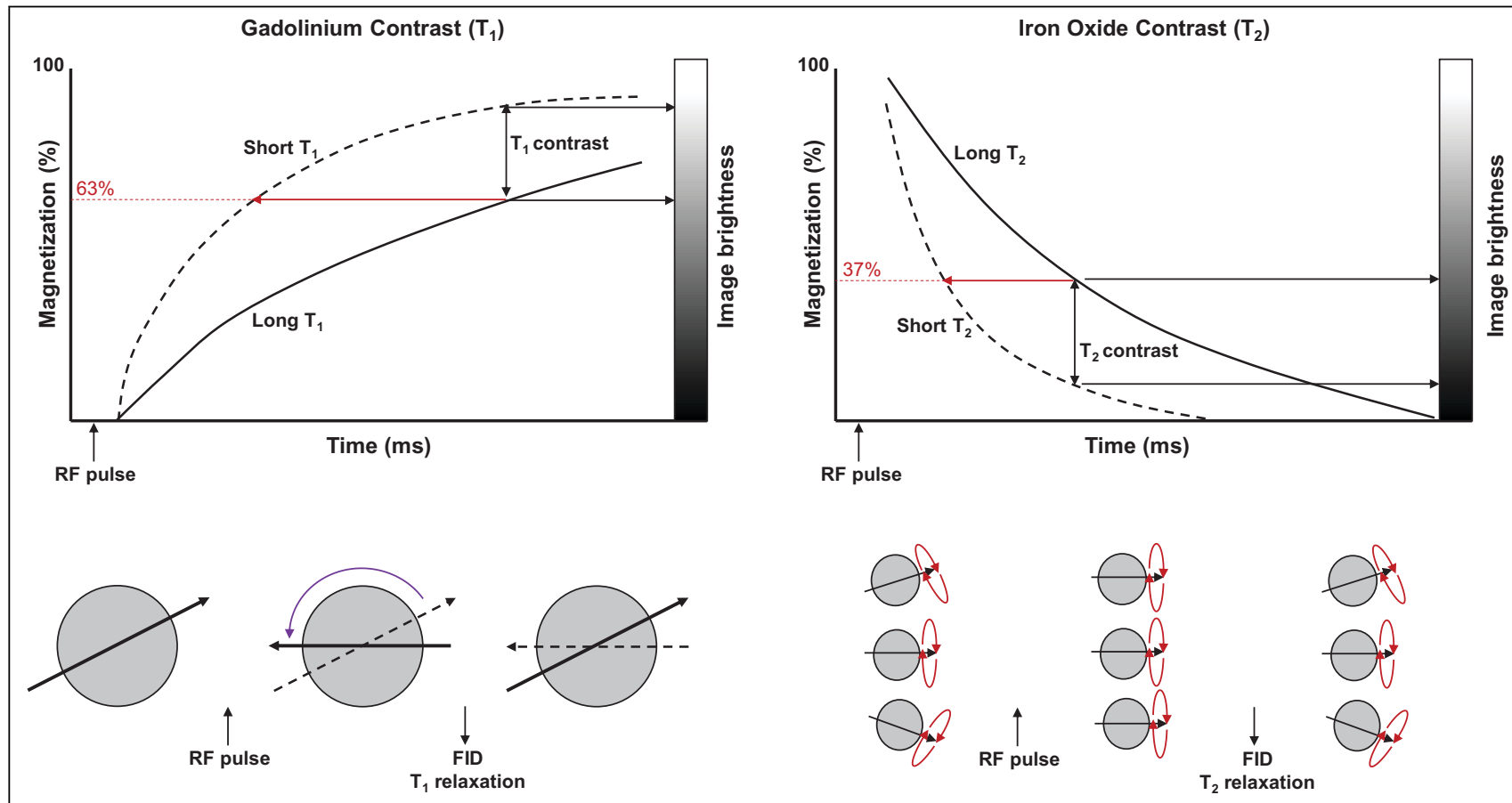

FIGURE 3. Schematic representation of principle of $T_{1}$ and $T_{2}$ contrast enhancement by altering relaxation times. $T_{1}$ plot (top left) shows effect of shortening relaxation time with gadolinium contrast and resultant positive enhancement of contrast. Likewise, $T_{2}$ plot (top right) shows effect of shortening relaxation time with iron oxide contrast and resultant negative enhancement of contrast. (Adapted with permission of (11).) RF = radiofrequency. 


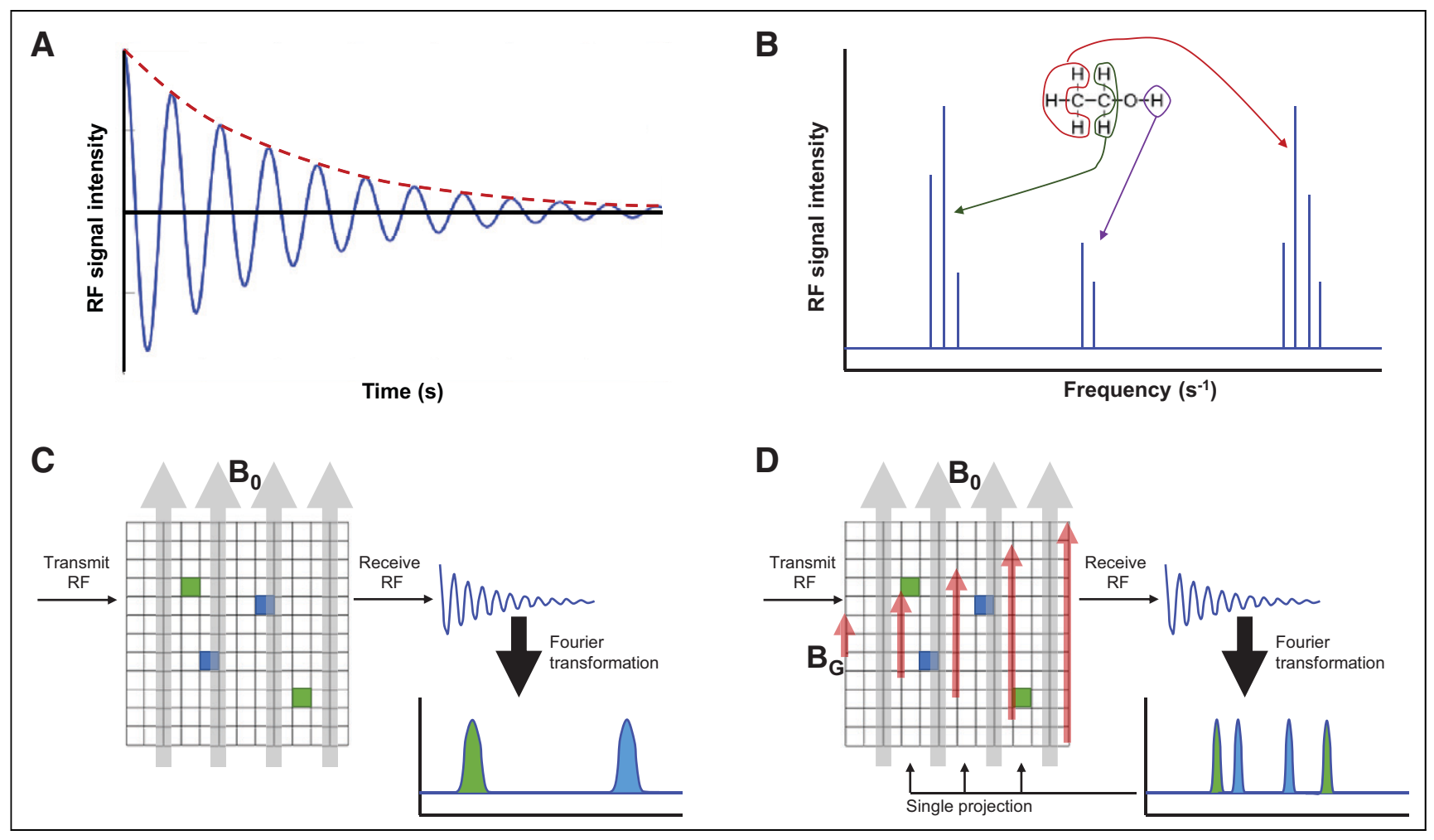

FIGURE 4. (A) FID with decreasing harmonic oscillation. (B) Fourier transformation of FID creates frequency-domain nuclear MR spectrum. For MRI, clusters represent different tissues, as is schematically represented here for nuclear MR spectroscopy, with each cluster representing a different molecular array of hydrogen (as depicted with red, green, and purple hydrogen atoms). (C) In static magnetic field $\left(B_{0}\right)$, nuclear MR spectrum identifies different tissues but not spatial location. (D) Application of gradient magnetic field ( $\left.B_{G}\right)$, depicted in red, allows spatial identification for that gradient projection. Rotating gradient magnetic field allows multiple projections to be collected and reconstructed. $\mathrm{RF}=$ radiofrequency.

(TR), and if sufficient time elapses between each radiofrequency pulse, equilibrium allows the amplitude of successive FIDs to be equal $(8,9)$. A short TR means equilibrium has not been reached and successive FIDs will have smaller amplitudes (partial saturation) $(8,9)$.

Alternatively, multiple pulse sequences can be used. If the $90^{\circ}$ radiofrequency pulse is followed by a $180^{\circ}$ radiofrequency pulse, an echo signal of the FID can be generated by the rephasing $(8,9)$. The echo signal is termed spin echo and is stronger than the FID signal; it is measured at the peak echo time $(8,9)$. Echo time after the $180^{\circ}$ radiofrequency pulse is equal to the time between the $90^{\circ}$ radiofrequency pulse and the $180^{\circ}$ radiofrequency pulse $(8,9)$. A multiecho spin-echo pulse sequence could be used in which a subsequent $180^{\circ}$ radiofrequency pulse follows the previous one, producing another spin echo $(8,9)$. An image can be generated from each of the spin echoes (primary and secondary) at the respective echo time to produce images of differing characteristics. The inversion-recovery pulse sequence starts with a $180^{\circ}$ radiofrequency pulse and follows it with a $90^{\circ}$ radiofrequency pulse that inverts the net magnetism $(8,9)$. There are a large variety of sequences used in MRI, and the details are beyond the scope of this article; however, those interested are encouraged to do further research on gradient echo sequences, dual-echo sequences, fluid-attenuated inversion-recovery sequences, diffusion-weighted sequences, short- $\tau$ inversion recovery sequences, and Dixon chemical shifts.

\section{PET/MR TECHNOLOGY}

\section{System Design}

One of the principal barriers to hybrid PET/MRI systems is the incompatibility of the technology. The traditional PET system design using photomultiplier tubes (PMTs) is susceptible to even small magnetic fields and, thus, incompatible with the large magnetic fields associated with MRI $(1,4-7)$. Furthermore, the electronics associated with signal processing in PET can create interference in the MR signal (4-7). This limitation gives rise to several solutions or configurations. The first is to have separate PET and MRI systems linked by a single patient table, with each gantry positioned and shielded sufficiently to not interfere with the other (e.g., Philips Ingenuity TF) (Fig. 5A). Alternatively, the detectors themselves could reside within the MRI system and be coupled by optical fibers to PMTs residing outside the magnetic field (Fig. 5B). This design may adopt a split-magnet design allowing the optical fibers to be shorter and the magnet bore wider. The genuinely integrated option would modify 


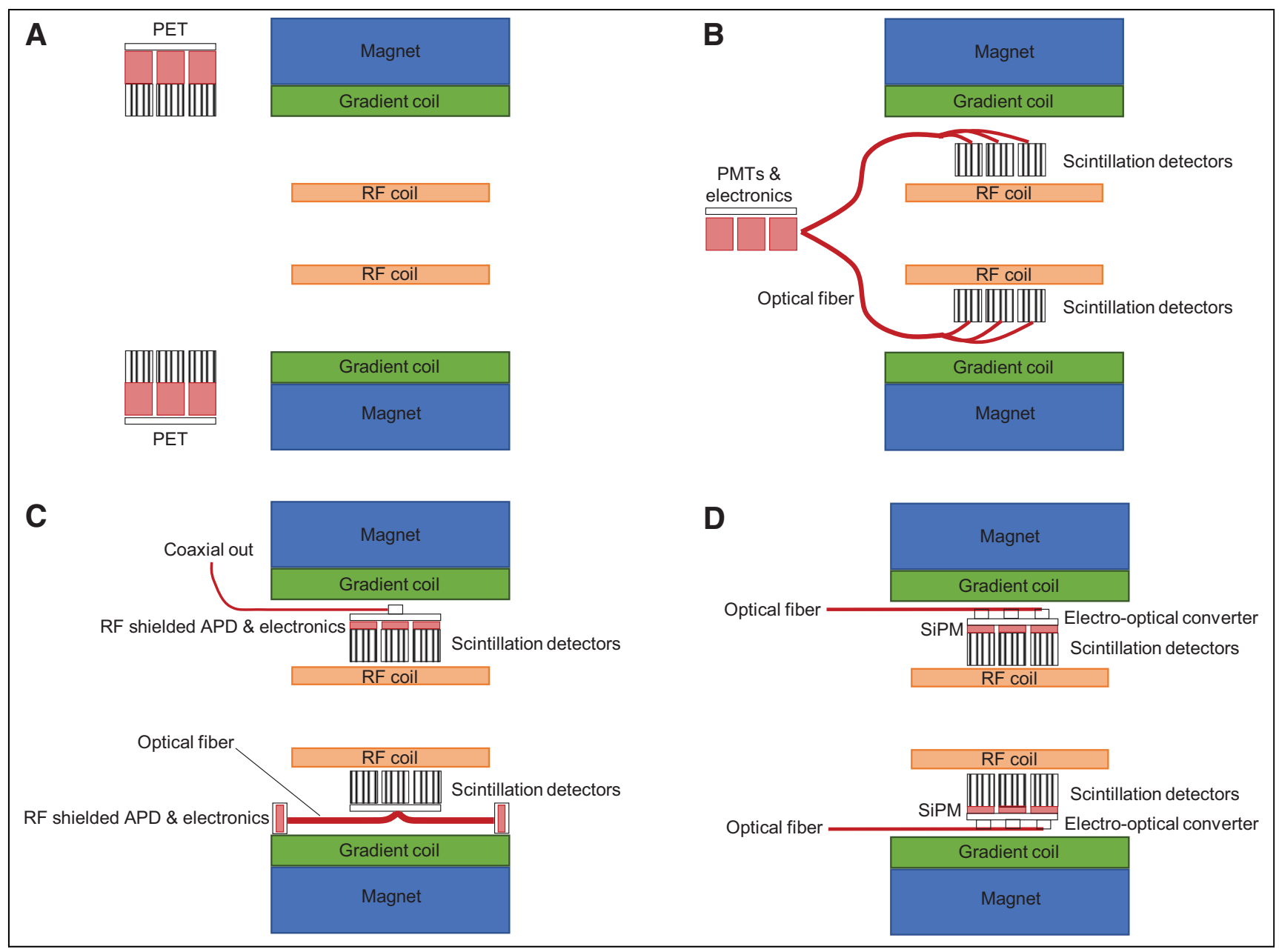

FIGURE 5. (A) Separate but linked PET/MR without option for simultaneous acquisition. (B) Integrated and simultaneous PET/MR housing radiofrequency (RF)-sensitive PMTs and electronics outside gantry. (C) Two versions of integrated PET/MR using RF-shielded APDs and electronics. Top half of schematic represents RF-shielded APD inside gantry, whereas bottom half uses optical fiber to connect to RF-shielded APDs at edge of MR field of view. (D) Integration of RF-compliant SiPM with optical fiber output obviates RF shielding of PET components.

detector composition to eliminate susceptibility from and to the magnetic field (e.g., Siemens Biograph mMR, GE Healthcare Signa PET/MR, and United Imaging uPMR 790 PET/ MR). Clearly, the integrated system is required to take advantage of simultaneous PET and MRI. One approach is to couple the scintillation detector directly or by optical fibers to avalanche photodiodes (APDs) in radiofrequency shielding (Fig. 5C). Alternatively, silicon photomultiplier (SiPM) detectors can be coupled to the scintillation detector (Fig. $5 \mathrm{D})$.

\section{Commercial Systems}

There are 4 commercially available clinical PET/MRI systems on the market. The Signa PET/MR is an integrated system with a 3-T MR component with PET (time of flight) that uses SiPM detectors directly coupled to lutetium-based crystals. The GE Healthcare MR750w wide-bore $(70 \mathrm{~cm})$ MRI system can be retrofitted with an SiPM PET insert to produce a 6-cm-bore PET/MR device. The Biograph mMR PET/MR is an integrated system with a 3-T MR component with PET that uses APD detectors and lutetium oxyorthosilicate crystals. The uPMR 790 PET/MR is an integrated system with a 3-T MR component with PET that uses SiPM detectors directly coupled to lutetium yttrium oxyorthosilicate (LYSO) crystals. The Ingenuity TF PET/MR is a sequential system with A 3-T MR component colocated and sharing an imaging system bed with a LYSO and PMT-based PET system.

\section{Detectors}

The traditional PET PMT was based on the coupling of bismuth germinate oxide scintillation crystal detectors to PMTs. More recently, the detectors have been upgraded to lutetiumbased scintillators such as lutetium oxyorthosilicate and LYSO. Lutetium oxyorthosilicate and LYSO have faster scintillation decay, which makes them more effective in high-count-rate conditions, in additional lines of response for 3-dimensional scanning, in longer axial fields of view, and in time of flight $(1,4,6,13)$. Lutetium oxyorthosilicate 
and LYSO have typically been coupled to PMTs. PMTs offer signal amplification but are susceptible to magnetic fields and are bulky, both being significant issues for integrating PET into an MRI gantry. APDs are silicon semiconductors that not only are able to operate within the magnetic field but also are compact $(1,4,6,7,13)$. Unfortunately APDs are thermally sensitive, which requires thermal stabilization. Furthermore, the signal gain is very low compared with a traditional PMT, which requires additional electronic signal amplification $(1,4,7,13)$. Both these requirements have been met successfully in traditional sequential PET/CT imaging system design and with some difficulties in PET/MR design. For example, the additional electronics for signal boosting is MR-sensitive, the thermal control creates bulk, and the low signal gain prohibits time of flight.
SiPMs are an alternative to the PMT and APDs, although technically, they are a type of APD $(1,7,13)$. SiPMs are a silicon semiconductor detector operating in Geiger mode and are also referred to in the literature as solid-state photomultipliers and multipixel photon counters (Fig. 6A) $(1,7,13)$. In the SiPM, the detector matrix comprises thousands of pixels that operate as a photon counter in Geiger mode. The pixels are known as singlephoton avalanche diodes, and each individual singlephoton avalanche diode can be counted independently and simultaneously with other single-photon avalanche diodes (Fig. 6B) (1,13). This approach results in high resolution, high sensitivity, high signal gain, low noise, fast timing, less magnetic susceptibility, and time-of-flight capability $(1,13)$.

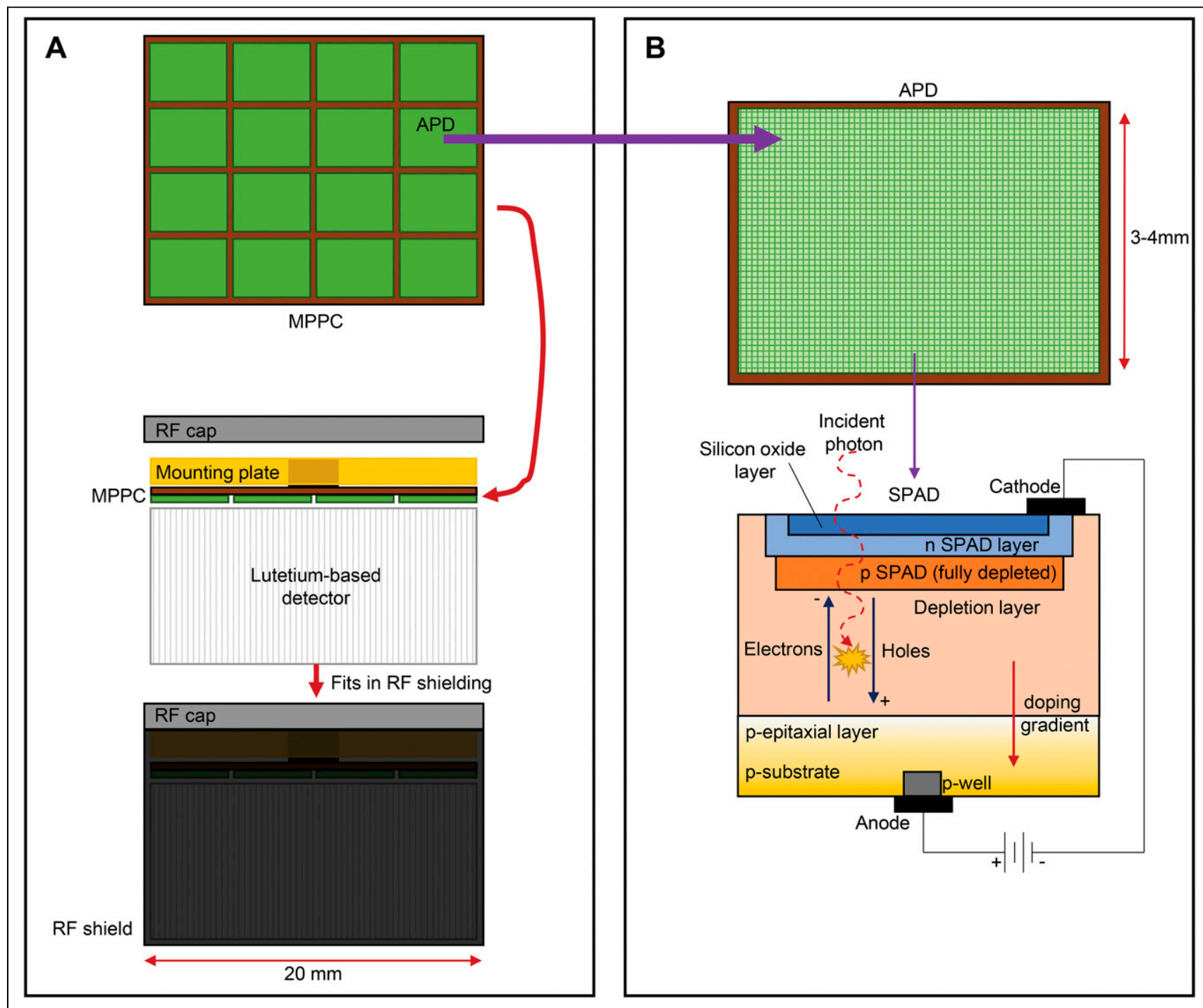

FIGURE 6. (A) Multipixel photon counter (MPPC) array comprises multiple APDs and is mounted to lutetium-based detector, which is housed in lightproof radiofrequency (RF) shield. These small individual MPPC units $(2 \mathrm{~cm})$ make up PET detector system. (B) APD (in this case, Geiger-mode APD) comprises pixels (single-photon avalanche diode, or SPAD). Layers of SPAD use silicon dioxide layer through which incident photons interact in depletion layer. Excitation causes electron holes that migrate to positive and negative doped layers to produce signal. 


\section{CHALLENGES OF PET/MR}

Even with the technical issues addressed, PET/MR comes with several challenges. Physical space, shielding, and planning are a major challenge but have been detailed in the first article in this series (14) and will not be duplicated here. The time cost associated with imaging protocols and procedures will only briefly be discussed below since it will be detailed in part 3 of this series of articles.

\section{Time Cost}

Although PET/MRI is considered simultaneous in nature, MRI radiofrequency-pulse sequences tend to be longer than PET bed positions (1). A 3-min bed position, for example, may be insufficient for all MRI sequences to be completed, although recent developments have seen the emergence of 4-5 MRI sequences in a 3-min PET bed position. This discrepancy is exacerbated in whole-body imaging typical of oncology studies. The solution under development is fast MRI protocols. These have shown some early success but have several barriers to overcome, including noise reduction. Nonetheless, multiple sequences for whole-body MRI over 20-40 min have a higher time cost than the multiple-bed-position PET covering the same anatomy in 10-20 min (1). An important consideration in this discussion is the actual applications of PET/MRI, which will be discussed in detail in part 4 of this series. Many of the clinical applications or clinical scenarios in which PET/MRI provides a tangible benefit over PET/CT may require only a single bed position - neurologic PET/MRI, cardiac PET/MRI, or head and neck oncology imaging, for example.

\section{Attenuation Correction}

A distinct advantage of PET/CT is the ability to perform accurate attenuation correction using a rapid $\mathrm{CT}$ acquisition immediately before or after the PET image is acquired. This was a significant advance over the previous methods using ${ }^{68} \mathrm{Ge} /{ }^{68} \mathrm{Ga}$ sources to produce transmission scans before the patient is administered the PET radionuclide dose. PET/ MRI has no option for producing a transmission scan for attenuation correction because there is no correlation between the attenuation coefficient and MR signal intensity $(1,5,6,15)$. A variety of approaches have been adopted to create an MR-based proxy for the attenuation map; each continues to be constrained by limitations. The challenge of accurate attenuation mapping using MRI is complicated by additional attenuation associated with MR hardware and coils in the field of view $(1,6)$. Furthermore, attenuating structures on the PET image may be positioned outside the acquisition field; an arms-down chest PET/MRI study, for example, may truncate the MR signal from the arms and, thus, be unable to estimate attenuation from the truncated tissues $(1,0)$. The advantage of PET/MRI attenuation correction over PET/CT, if it can be performed accurately, is the reduced patient radiation dose and the simultaneous acquisition that can overcome mismatches between sequentially performed PET and CT attenuation mapping (1).
Simple segmentation and classification of different tissue types using the $T_{1}$ signal can be constrained by the lack of bone signal and associated attenuation correction $(1,5,7,15)$. Moreover, some tissues, such as lung, can have different attenuation coefficients from one person to the next (1). Another simple approach may be suitable for tissues with uniform attenuation (e.g., the brain) using estimation methods and an attenuation formula (1). This method was used for brains before PET/CT, but the additional influence of MRI coils requires careful mapping of the coil template in the estimated attenuation corrections $(1,15)$. It is possible to also revert to previous PET system designs in which a rotating rod source $\left({ }^{68} \mathrm{Ge} /{ }^{68} \mathrm{Ga}\right)$ is used for a transmission scan $(1,15)$. Obviously, this approach adds to the time associated with the transmission scan, produces noisy attenuation maps, and remains inferior to CT-based attenuation correction.

Current approaches to attenuation correction on integrated PET/MRI use a 3-dimensional Dixon approach that provides in-phase and out-of-phase datasets for water and fat (6). In essence, this allows segmentation of air, fat, muscle, and lung tissue for attenuation coefficients but lacks accommodation for bone (6). Bone is classified as soft tissue from an attenuation coefficient perspective in this approach. The underestimation of attenuation associated with this bone nonclassification can produce substantial errors in quantitation of both bone and adjacent soft tissue. This could be overcome with the addition of atlas-based methods, but these have limitations around variations from normal anatomy not uncommon in pathologic patients (15). A different approach incorporates ultrashort-echo signals to delineate bone and add to the standard $\mathrm{T}_{1}$ tissue segmentation. The ultrashortecho-time approach can also be combined with a Dixon sequence (MRI sequence based on chemical shift designed for fat suppression) to produce classes of very short $T_{2}$ tissues for segmentation $(5,6,15)$. This approach has particular potential in lung imaging.

An important area of development for PET/MRI is pseudoCT attenuation maps (Fig. 7). There are several limitations in estimating an attenuation map from MRI for PET/MRI that convolutional neural networks (CNNs) may overcome (16). Deep CNNs can produce an attenuation map that closely models the CT-based grounded truth $(17,18)$. Others have integrated the Dixon method with a CNN to generate pseudo-CT for pelvic PET/MRI, with promising results of less than $2 \%$ variation from the CT map (19). More accurate attenuation maps also resulted from the use of deep CNN combined with zero-echo-time Dixon pseudo-CT (20). $\mathrm{CNN}$ approaches can produce pseudo-CT attenuation maps from the PET sinogram, and this approach was used successfully $\left(<1 \%\right.$ error) on ${ }^{18} \mathrm{~F}$-FDG brain scans $(21)$.

The ability to accurately correct for attenuation is essential for accurate quantitation of PET data $(1,6)$. Indeed, the accuracy of relative quantitation markers such as SUV will be undermined if the integrity of attenuation correction is sacrificed. 


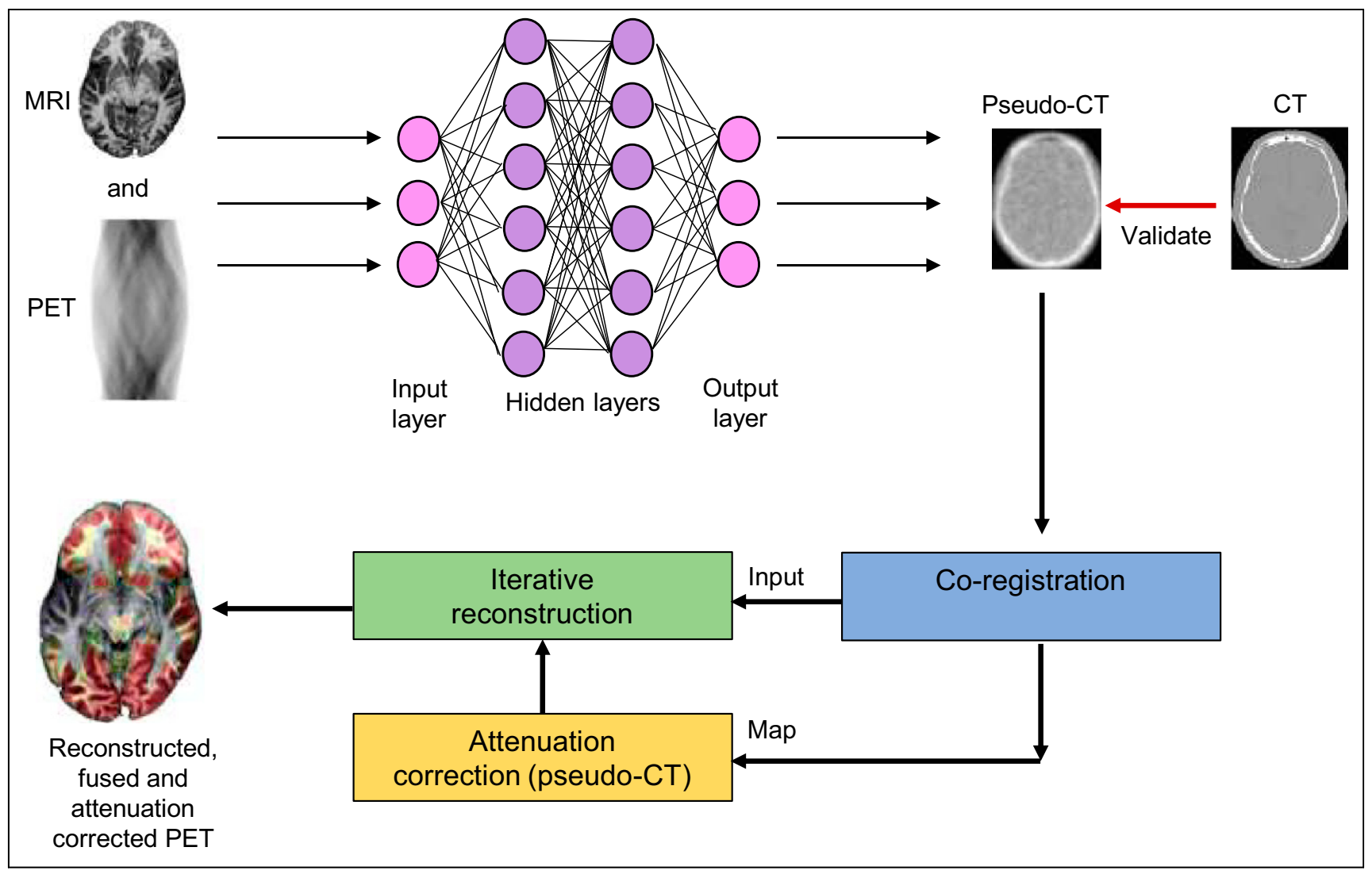

FIGURE 7. Model for potentially using CNN for improved pseudo-CT attenuation correction in PET/MRI. (Adapted from (16).)

\section{Artifacts}

Both PET and MRI are vulnerable to artifacts, and this vulnerability can undermine not only image quality but also quantitative accuracy. PET/MRI, combined, adds a layer of complexity to potential artifacts. The most common artifact that raises concern is cross-talk between PET and MRI, but this has been largely overcome by the new detector designed discussed previously. It is possible that mechanical misalignment can cause coregistration inaccuracies (6). Imperfect attenuation correction remains a concern for artifact production, and this can be exacerbated by the administration of contrast by interfering with tissue segmentation (6). MRI is susceptible to signal voids associated with items such as implants, and these may also produce attenuation artifacts on the PET scan (6). As already discussed, artifacts can be produced in the PET data by attenuation from the MRI hardware and coils and by truncation of the field of view.

\section{CONCLUSION}

Although hybrid imaging with PET/MR plays a role in several clinical situations, there are several technical considerations required in the design and operation of the imaging systems. Specifically, modifications to conventional imaging systems that accommodate integration of the 2 modalities without image-degrading cross talk require deep understanding before the technology can be adopted. Such understanding will have translational benefits to procedural and clinical applications of PET/MRI. Specific design features of PET/MRI help guide the facility planning detailed in part 1 of this series. The integration of PET/MRI affords the opportunity for enhanced protocols and clinical applications which will be explored in more detail in parts 3 and 4 of this series, respectively. Understanding the principles of PET, MRI, and integrated PET/MRI systems provides the foundation for safe, effective, and optimal adoption of PET/MR technology.

\section{DISCLOSURE}

No potential conflict of interest relevant to this article was reported.

\section{REFERENCES}

1. Vandenberghe S, Marsden PK. PET-MRI: a review of challenges and solutions in the development of integrated multimodality imaging. Phys Med Biol. 2015;60:R115R154.

2. Fendler WP, Czernin J, Herrman K, Beyer T. Variations in PET/MRI operations: results from an international survey among 39 active sites. $J$ Nucl Med. 2016;57: 2016-2021.

3. Turkington TG. Introduction to PET instrumentation. J Nucl Med Technol. 2001;29: 4-11.

4. Wehrl HF, Sauter AW, Judenhofer MS, Pichler BJ. Combined PET/MR imaging: technology and applications. Technol Cancer Res Treat. 2010;9:5-20.

5. Herzog H. PET/MRI: challenges, solutions and perspectives. Z Med Phys. 2012;22: 281-298.

6. Quick HH. Integrated PET/MR. J Magn Reson Imaging. 2014;39:243-258. 
7. Disselhorst JA, Bezrukov I, Kolb A, Parl C, Pichler BJ. Principles of PET/MR imaging. J Nucl Med. 2014;55(suppl):2S-10S.

8. Bushong SC, Clarke G. Magnetic Resonance Imaging: Physical Principles and Biological Principles. 4th ed. Elsevier; 2015.

9. Hashemi RH, Bradley WG, Lisanti CJ. MRI: The Basics. 2nd ed. Lippincott Williams \& Wilkins; 2004.

10. van Geuns RJM, Wielopolski PA, de Bruin HG, et al. Basic principles of magnetic resonance imaging. Prog Cardiovasc Dis. 1999;42:149-156.

11. Currie GM. Pharmacology, part 5: CT and MRI contrast media. J Nucl Med Technol. 2019;47:189-202.

12. Currie G, Hewis J, Bushong S. Tomographic reconstruction; a non-mathematical overview. J Med Imaging Radiat Sci. 2015;46:403-412.

13. Gundacker $\mathrm{S}$, Heering $\mathrm{A}$. The silicon photomultiplier: fundamentals and applications of a modern solid-state photon detector. Phys Med Biol. 2020;65:17TR01

14. Kamvosoulis P, Currie G. PET/MR, part 1: establishing a PET/MRI facility. J Nucl Med Technol. 2021;49:120-125.

15. Martinez-Möller A, Nekolla SG. Attenuation correction for PET/MR: problems, novel approaches and practical solutions. Z Med Phys. 2012;22:299-310.
16. Currie GM. Intelligent imaging: artificial intelligence augmented nuclear medicine. $J$ Nucl Med Technol. 2019;47:217-222.

17. Hwang D, Kim KY, Kang SK, et al. Improving the accuracy of simultaneously reconstructed activity and attenuation maps using deep learning. $J$ Nucl Med. 2018;59: 1624-1629.

18. Hwang D, Kang SK, Kim KY, et al. Generation of PET attenuation map for wholebody time-of-flight 18F-FDG PET/MRI using a deep neural network trained with simultaneously reconstructed activity and attenuation maps. J Nucl Med. 2019;60: 1183-1189.

19. Torrado-Carvajal A, Vera-Olmos J, Izquierdo-Garcia D, et al. Dixon-VIBE Deep Learning (DIVIDE) pseudo-CT synthesis for pelvis PET/MR attenuation correction. J Nucl Med. 2019;60:429-435.

20. Leynes AP, Yang J, Wiesinger F, et al. Zero-Echo-Time and Dixon Deep Pseudo-CT (ZeDD CT): direct generation of pseudo-CT images for pelvic PET/MRI attenuation correction using deep convolutional neural networks with multiparametric MRI. $J$ Nucl Med. 2018;59:852-858.

21. Liu F, Jang H, Kijowski R, Zhao G, Bradshaw T, McMillan AB. A deep learning approach for ${ }^{18}$ F-FDG PET attenuation correction. EJNMMI Phys. 2018;5:24. 